\title{
Avaliação de procedimentos para identificar mortes maternas
}

\author{
Evaluation of procedures \\ for identifying maternal deaths
}

Sandra Valongueiro ${ }^{1}$

Ana Bernarda Ludermir 2

Lílian Albuquerque Ferraz Gominho 3

\footnotetext{
1 Núcleo de Saúde Pública, Universidade Federal de Pernambuco. Av. Professor Moraes Rego $s / n$, Bloco E, 4o andar, Cidade Universitária, Recife, $P E$ 50670-901, Brasil. nusp@nusp.ufpe.br 2 Departamento de Medicina Social, Centro de Ciências da Saúde, Universidade Federal de Pernambuco. Av. Professor Moraes Rego s/n, Bloco D, lo andar, Cidade Universitária, Recife, $P E$ 50670-901, Brasil. 3 Departamento de Epidemiologia Secretaria Municipal de Camaragibe. Av. Belmiro Correia 2340 Camaragibe, $P E$ 54768-000, Brasil.
}

\begin{abstract}
The main problems identified during maternal mortality surveillance in Pernambuco State, Brazil, were low coverage and poor quality of data, as well as the inadequacy of data-collection instruments. Based on these findings, a new instrument called the Confidential Form for Reporting Deaths of Reproductive-Age Women was developed, and later an evaluation was conducted on the available procedures for distinguishing maternal deaths among female deaths. The RAMOS method, using the new questionnaire, was applied in Camaragibe (a city in Greater Metropolitan Recife) based on the female deaths recorded in 2000. According to the results, this method, using the new form, showed improved recording of socio-demographic and female mortality variables as compared to both the maternal mortality surveillance conducted by the Municipal Epidemiological Department and the death certificate system. This finding reinforces the need to improve maternal mortality surveillance in the study area, taking into account the RAMOS method and the new instrument.
\end{abstract}

Key words Evaluation; Maternal Mortality; Mortality Registries

Resumo Os principais problemas identificados no sistema de vigilância do óbito materno em Pernambuco, Brasil, foram a baixa cobertura e qualidade das informações e a inadequação do instrumento de notificação. Com base nesta crítica, decidiu-se elaborar uma nova ficha de notificação chamada Ficha Confidencial de Notificação de Mortes de Mulheres em Idade Fértil, $e$ em seguida avaliar os procedimentos disponíveis para identificar óbitos maternos entre os óbitos femininos. O método RAMOS, usando a nova ficha de notificação, foi conduzido em Camaragibe, município da Região Metropolitana do Recife, a partir dos óbitos femininos registrados em 2000. Os resultados mostraram que este método foi capaz de obter mais informações sobre variáveis sócio-demográficas e sobre variáveis diretamente relacionadas com o óbito feminino do que a investigação de rotina conduzida pelo município, e do que a declaração de óbito. Estes achados reforçam a necessidade de se implementar a vigilância do óbito materno na região metropolitana, tendo-se como referência o método RAMOS e a nova ficha de notificação.

Palavras-chave Avaliação; Mortalidade Materna; Registros de Mortalidade 


\section{Introdução}

Algumas pesquisas têm demonstrado que o nível de subinformação da mortalidade materna no Brasil é de aproximadamente 50\% (Laurenti et al., 1990; Tanaka \& Mitsuiki, 1999; Volochko, 1992). As regiões Norte e Nordeste do Brasil são responsáveis pelos maiores índices de sub-registro nos sistemas de informações de mortalidade (estatísticas do registro civil da Fundação Instituto Brasileiro de Geografia e Estatística - IBGE - e estatísticas de mortalidade do Ministério da Saúde - MS), embora exista uma certa precariedade nas informações sobre mortes maternas e infantis em todas as regiões brasileiras.

Em Pernambuco, desde o início da década de 90, são observados esforços no sentido de implementar os sistemas de informações em saúde (Sistema de Informação sobre Mortalidade - SIM; Sistema de Informação sobre Nascidos Vivos - SINASC; e Sistema de Notificação sobre Agravos Notificáveis - SINAN), entretanto, elevados níveis de sub-registro e subinformação ainda são identificados, tanto em relação a óbitos infantis, como a óbitos maternos. A Secretaria Estadual de Saúde de Pernambuco (SES-PE), na perspectiva de intervir diretamente sobre a subinformação de óbitos maternos, definiu por meio de Portaria Estadual (SES$\mathrm{PE}, 1995)$, que todas as mortes de mulheres em idade reprodutiva (entre 10 e 49 anos) deveriam ser investigadas pelos departamentos de epidemiologia dos municípios. Nesse sentido, em 1996, técnicos de epidemiologia de $90 \%$ dos municípios do estado foram capacitados em investigação de óbitos maternos sob a coordenação da antiga Divisão de Saúde da Mulher e Adolescente e do Departamento de Informações da SES-PE, com o objetivo de implantar um sistema de vigilância do óbito materno (SES-PE, 1999).

Durante monitoramento do sistema de vigilância do óbito materno implantado no estado desde 1997, foram identificados problemas na cobertura e qualidade das informações obtidas com a investigação de rotina. A elevada rotatividade de profissionais do setor de epidemiologia e conflitos na eleição de prioridades pelos gestores municipais foram tidos como alguns dos elementos responsáveis pela precariedade dos dados colhidos pelos municípios.

Os instrumentos (fichas) utilizados também foram considerados inadequados à notificação e investigação dos óbitos de mulheres em idade fértil. O instrumento até então utilizado fora construído pelo MS no início dos anos 90, e mesmo modificado em 1997, não conseguiu incorporar as especificidades das investigações realizadas em hospitais e domicílios. Embora não exista instrumento perfeito, um instrumento deve ser adequado à população em termos de tempo, custo e linguagem. Considerando essa crítica, foi elaborado pela investigadora principal um novo instrumento, baseado em instrumentos utilizados por outras instituições / comitês de mortes maternas e infantis e na técnica de autópsia verbal (WHO, 1994), composto de três fichas: Ficha Confidencial de Notificação de Óbitos de Mulheres em Idade Fértil, Ficha Confidencial de Investigação de Óbitos $\mathrm{Ma}$ ternos e Ficha Confidencial de Entrevista Domiciliar.

Este estudo avalia procedimentos para identificar mortes maternas no Município de Camaragibe, Região Metropolitana do Recife, em 2000, usando-se diferentes instrumentos de coleta de dados: a Ficha Confidencial de Notificação de Óbitos de Mulheres em Idade Fértil, a ficha antiga de notificação e a declaração de óbito.

\section{Conceitos e definições}

Este estudo utilizou a definição de morte materna precoce e tardia da Classificação Internacional de Doenças - CID 10a Revisão (OMS, 1994), e embora existam vantagens no uso da classificação de morte por causa múltipla (Nam, 1990), este estudo utilizará o conceito de "causa básica de morte” para classificar as mortes maternas. Outras definições utilizadas foram as mortes maternas presumíveis e as mortes maternas descartadas. Uma morte feminina foi considerada morte materna presumível quando a causa de morte registrada no certificado de óbito de mulheres em idade reprodutiva não representou a causa básica, mas uma causa associada ou causa terminal (MS, 2001). Neste estudo todas as mortes femininas entre 10 e 49 anos foram consideradas como mortes maternas presumíveis. Uma morte materna foi considerada descartada, quando após a investigação, foi definida como não materna. Apenas mortes femininas investigadas foram consideradas descartadas neste estudo. Seguindo ainda a necessidade de esclarecer os conceitos aqui utilizados, sub-registro representou os óbitos não registrados em cartório e subinformação ou subnotificação representaram as causas de morte não informadas ou omitidas no atestado médico das declarações de óbito. 


\section{Método}

\section{Área de estudo}

Camaragibe é um município da Região Metropolitana do Recife, cuja população em 2000 foi de 128.627 habitantes (IBGE, 2001), sendo $48.6 \%$ homens e $51.4 \%$ mulheres. Possui uma área de $52,9 \mathrm{~km}^{2}$ e está dividida em cinco regiões político-adminstrativas: Timbi, Alberto Maia, Primavera, Tabatinga e Aldeia. Embora Alberto Maia e Aldeia tenham características rurais, o município como um todo é classificado como região urbana pelo IBGE.

Camaragibe está sob regime de municipalização plena, cujo modelo de assistência à saúde tem os agentes comunitários e as unidades de saúde da família como portas de entrada do sistema. Dispunha em 2000 de 32 unidades do Programa Saúde da Família (USF), dois centros de saúde, dois centros de especialidades, três hospitais privados contratados pelo SUS e outros serviços especializados como laboratórios e centro de diagnósticos, e uma maternidade sendo equipada (Prefeitura Municipal de Camaragibe, 2001). Segundo o Departamento de Epidemiologia do município, a taxa de mortalidade infantil caiu de 65,1/1.000 nascidos vivos em 1993 para 15,5/1.000 nascidos vivos em 2000. Por outro lado, a razão de mortalidade materna estimada foi $80 / 100$ mil nascidos vivos para o período 1998/2000, entretanto, como não existiam dados confiáveis sobre mortalidade materna para períodos anteriores, não foi possível avaliar sua tendência. Como em Camaragibe, todos os óbitos de mulheres em idade fértil (10 a 49 anos) estão sendo investigados desde 1998, este município representou um ótimo campo para a condução da etapa inicial da validação do instrumento de morte materna. As mortes de mulheres em idade fértil representaram $19,3 \%$ do total de mortes femininas (324) entre as 814 registradas no município (Prefeitura Municipal de Camaragibe, 2001).

Não há registros de cemitérios clandestinos no município e os óbitos de mulheres residentes em outros municípios ocorridos em Camaragibe não foram considerados como população de estudo.

\section{Desenho e população de estudo}

Um estudo descritivo foi conduzido durante os meses de julho, agosto e setembro de 2001 em Camaragibe, com o objetivo de avaliar procedimentos para identificar mortes maternas, usando-se diferentes instrumentos de coleta de informações, uma nova Ficha Confidencial de Notificação de Óbitos de Mulheres em Idade Fértil, a ficha antiga de notificação e a declaração de óbito. Dois passos compunham inicialmente o processo de avaliação: a reinvestigação de todos os óbitos femininos registrados em Camaragibe em 2000 com o Método RAMOS (Reproductive Age Mortality Study) e a comparação das informações obtidas por meio dos diferentes procedimentos e instrumentos.

Embora o Departamento de Epidemiologia deste município tenha em princípio considerado que todas as mortes de mulheres em idade fértil foram investigadas com a ficha antiga de notificação, das 64 mortes registradas, apenas 44 foram investigadas previamente (69\%). Na perspectiva de minimizar o custo/benefício das investigações, a equipe da Secretaria Municipal da Saúde de Camaragibe (SMS-Camaragibe), assumiu como verdadeiras as 18 respostas negativas às variáveis da declaração de óbito que contêm as seguintes perguntas: "a mulher estava grávida no momento da morte? A mulher esteve grávida nos doze meses anteriores à morte?", cujas respostas possíveis seriam Sim, Não ou Ignorado. Em dois casos, a ficha antiga não foi usada porque as mulheres tinham identidades desconhecidas naquela época e os domicílios não foram localizados. Com base nestas informações, sentiu-se a necessidade de redimensionar as etapas do estudo. Na primeira, 44 mortes femininas previamente investigadas pela SMS-Camaragibe com a ficha antiga foram reinvestigadas com Ficha Confidencial de Notificação de Óbitos de Mulheres em Idade Fértil, usando-se o método RAMOS. Em seguida, foram investigadas as vinte mortes femininas não investigadas previamente.

As informações relativas às mortes de mulheres em idade fértil foram reclassificadas como: morte materna declarada, se a mesma fora previamente classificada como morte materna na declaração de óbito; morte materna presumível, se associada à gravidez, mesmo que não classificada como morte materna na declaração de óbito e morte materna descartada, se após uma investigação preliminar, a causa materna fora excluída.

$\underline{\text { Instrumentos e procedimentos }}$

A Ficha Confidencial de Notificação de Óbitos de Mulheres em Idade Fértil é a primeira ficha de um instrumento formado por ela e pelas $\mathrm{Fi}$ cha Confidencial de Investigação de Óbitos Maternos e Ficha Confidencial de Entrevista Domiciliar. É composta por perguntas fechadas, se- 
mi-abertas e abertas e dividida em cinco seções, como pode ser vista em anexo. A seção inicial (0) diz respeito aos dados gerais da notificação, a seção um (1) aos dados sócio-demográficos (padronizados pela versão da declaração de óbito em vigor), a dois (2) aos dados do óbito, a três (3) às classificações da causa de morte presentes na declaração de óbito e a seção quatro (4) ao tipo de óbito feminino após a investigação inicial (se declarado, presumível ou descartado). Esta seção contém ainda espaço para observações do (a) investigador (a).

A Ficha de Notificação de Óbito de Mulher em Idade Fértil (ficha antiga) também é composta por perguntas fechadas, semifechadas e abertas, embora contenha uma maior proporção de perguntas abertas. É composta por quatro seções e as variáveis sócio-demográficas não foram padronizadas pela declaração de óbito, e também há pergunta sobre chefia de domicílio. As variáveis relacionadas com o momento da morte são baseadas na CID - 9a Revisão, portanto desconsidera o puerpério tardio. Por fim, a ficha antiga de notificação não contém uma seção para observações do (a) investigador (a).

O método RAMOS envolve a identificação e a investigação de causas de óbitos de mulheres em idade fértil e, embora possa gerar informações incompletas, vem sendo usado com sucesso em países ou regiões com precário sistema de informações sobre mortes maternas e infantis como a Jamaica (1986), o Egito (1990) e Honduras (1990). No Brasil, este método foi inicialmente usado por Laurenti et al. (1990), no Município de São Paulo e posteriormente por Tanaka \& Mitsuiki (1999). A Organização Mundial da Saúde (OMS) e o Fundo das Nações Unidas para a Infância (UNICEF), o consideram como o padrão ouro para estimar mortalidade materna (WHO, 2001). Em Pernambuco, ele foi utilizado por Albuquerque (1994) e Valongueiro (1996), para estimar mortalidade materna na Cidade do Recife e em quatro regiões do estado.

\section{Coleta de dados}

Os óbitos das mulheres em idade fértil registrados no período e local de referência, foram selecionados com base nas declarações de óbito obtidas no arquivo do Departamento de Epidemiologia da SMS-Camaragibe. O Programa Saúde da Família (PSF) e a mídia representaram fontes adicionais de notificação dos óbitos femininos.

A Ficha Confidencial de Notificação de Óbitos de Mulheres em Idade Fértil (ficha nova) foi cuidadosamente preenchida com informações obtidas nos locais de ocorrência, hospitais e domicílios, serviços de necropsias e PSF. As variáveis sócio-demográficas e as relativas à causa do óbito obtidas na declaração de óbito e nas outras fontes de notificação, foram confirmadas ou resgatadas dos prontuários médicos, livros de registros de parto, entrevistas com profissionais de saúde e/ou informações de familiares. Os (as) investigadores (as) foram pessoas treinadas no SIM.

As fichas antigas de notificação também foram obtidas no arquivo do Departamento de Epidemiologia da SMS-Camaragibe, mas desconsideradas nesta etapa da coleta de dados.

\section{Análise dos dados}

O banco de dados contendo informações dos três instrumentos, Ficha Confidencial de Notificação de Óbitos de Mulheres em Idade Fértil, ficha antiga de notificação e declaração de óbito, foi estruturado no SPSS.

A comparação entre os três instrumentos se deu por meio das razões de proporções do grau de preenchimento das variáveis sócio-demográficas (idade, escolaridade, situação conjugal, raça/cor e ocupação) e daquelas relacionadas diretamente com a morte (local de ocorrência, momento da morte e classificação do óbito feminino).

Com o objetivo de explorar todas as informações obtidas durante a investigação dos óbitos, uma segunda série de comparações foi realizada na qual foram incorporados todos os óbitos de mulheres em idade fértil (64) reinvestigados. Neste sentido, compararam-se as informações obtidas com o método RAMOS, usando-se a ficha nova, com as informações registradas no SIM com base na declaração de óbito (acrescentando-se os vinte óbitos não investigados pela rotina do Departamento de Epidemiologia do município).

\section{Resultados}

Das 44 mortes femininas reinvestigadas, 35 $(79,5 \%)$ foram descartadas como morte materna. Entre as quatro mortes maternas declaradas $(9 \%)$, todas mantiveram a classificação anterior, posteriormente confirmada por meio das outras fichas que integram o procedimento, a Ficha Confidencial de Investigação de Morte Materna e a Ficha Confidencial de Entrevista Domiciliar (não discutidas neste artigo).

As variáveis sócio-demográficas (Tabela 1), foram em geral melhor preenchidas durante a 
Grau de preenchimento das variáveis sócio-demográficas comuns à Ficha Confidencial de Notificação de Ó bitos de Mulheres em Idade Fértil (ficha nova), obtida por meio da reinvestigação, à ficha antiga de notificação obtida por meio da investigação de rotina e à declaração de óbito. Camaragibe, Pernambuco, Brasil, 2000*.

\begin{tabular}{|c|c|c|c|c|c|c|c|c|c|c|}
\hline \multirow{2}{*}{$\begin{array}{l}\text { Procedimentos de investigação/ } \\
\text { proporções }\end{array}$} & \multicolumn{2}{|c|}{ Idade } & \multicolumn{2}{|c|}{ Raça/Cor } & \multicolumn{2}{|c|}{ Escolaridade } & \multicolumn{2}{|c|}{ Situação conjugal } & \multicolumn{2}{|c|}{ O cupação } \\
\hline & $\mathrm{n}$ & $\%$ & $\mathrm{n}$ & $\%$ & $\mathrm{n}$ & $\%$ & $\mathrm{n}$ & $\%$ & $\mathrm{n}$ & $\%$ \\
\hline Ficha nova (a) & 44 & 100,0 & 44 & 100,0 & 43 & 97,7 & 44 & 100,0 & 38 & 88,6 \\
\hline Ficha antiga (b) & 42 & 95,5 & 38 & 88,0 & 33 & 75,0 & 43 & 97,7 & 32 & 72,7 \\
\hline Declaração de óbito (c) & 44 & 100,0 & 22 & 50,0 & 7 & 15,9 & 37 & 84,0 & 32 & 72,7 \\
\hline Razão (a/b) & \multicolumn{2}{|c|}{1,05} & \multicolumn{2}{|c|}{1,10} & \multicolumn{2}{|c|}{1,30} & \multicolumn{2}{|c|}{1,10} & \multicolumn{2}{|c|}{1,20} \\
\hline Razão (a/c) & \multicolumn{2}{|c|}{1,05} & \multicolumn{2}{|c|}{1,70} & \multicolumn{2}{|c|}{6,14} & \multicolumn{2}{|c|}{1,20} & \multicolumn{2}{|c|}{1,20} \\
\hline Razão (b/c) & \multicolumn{2}{|c|}{0,96} & \multicolumn{2}{|c|}{1,50} & \multicolumn{2}{|c|}{4,72} & \multicolumn{2}{|c|}{1,10} & \multicolumn{2}{|c|}{1,00} \\
\hline
\end{tabular}

* Três casos de morte materna ocorreram fora de 2000.

reinvestigação usando-se a ficha nova do que com a investigação prévia, usando-se a ficha antiga e a declaração de óbito registrada pelo SIM. A variável escolaridade chegou a ser seis vezes mais preenchida com a reinvestigação do que com a declaração de óbito. A variável ocupação foi a que apresentou a menor variação, observando-se que a proporção de preenchimento obtida foi a mesma tanto com a investigação prévia, usando-se a ficha antiga, como na ausência de investigação, quando apenas a declaração de óbito foi considerada $(72,7 \%)$.

A reinvestigação usando-se o método RAMOS demonstrou melhor preenchimento das variáveis relacionadas com a morte feminina. Vale ressaltar que a proporção obtida com a investigação anterior foi melhor do que os dados registrados pelo SIM, enquanto o local de ocorrência do óbito não apresentou variações (97\%). A reinvestigação mostrou $100 \%$ de preenchimento desta variável. A maior diferença, entretanto, se deu em relação à classificação do óbito feminino: a reinvestigação classificou $50 \%$ mais óbitos femininos como maternos do que a investigação prévia, e duas vezes mais do que as informações geradas pelo SIM via declaração de óbito (Tabela 2).

As Tabelas 3 e 4 apresentam os resultados das comparações realizadas entre as informações obtidas com o método RAMOS, usandose a ficha nova, com as informações registradas no SIM com base na declaração de óbito (64 mortes femininas).

As variáveis sócio-demográficas foram, com algumas exceções, melhor preenchidas durante a reinvestigação. A reinvestigação foi capaz de registrar aproximadamente duas vezes mais as variáveis raça/cor e escolaridade do que o
SIM; ocupação apresentou a mesma proporção de preenchimento, independentemente do procedimento utilizado na investigação. A variável idade, no entanto, foi melhor registrada pelo SIM do que pela reinvestigação. Como a variável data de nascimento esteve presente apenas na declaração de óbito, esta informação pode ter servido de base para o cálculo da idade e assim aumentando seu grau de preenchimento.

As variáveis diretamente relacionadas com a causa da morte (momento da morte e classificação do óbito feminino) foram mais bem registradas com a reinvestigação. E considerando-se o grau de preenchimento da classificação do óbito feminino como uma estimativa da cobertura da mortalidade materna no município, a reinvestigação com o método RAMOS identificou mais que o dobro de mortes maternas em relação aos óbitos declarados pelo SIM (10 vs. 4), enquanto a investigação de rotina conduzida usando-se a ficha antiga detectou apenas seis mortes maternas (10 vs. 6), o que representa fatores de correção da ordem de 2,5 e 1,5, respectivamente.

\section{Discussão}

A despeito das limitações decorrentes do reduzido número de óbitos, a avaliação demonstrou que o método RAMOS foi o melhor procedimento para identificar mortes maternas em Camaragibe. Embora as comparações tenham sido conduzidas com três instrumentos diferentes, o mecanismo usado para identificar os óbitos femininos, usando-se a Ficha Confidencial de Notificação de Óbitos de Mulheres em Idade Fértil parece ter sido um facilitador para a coleta de dados. 
Tabela 2

Grau de preenchimento das variáveis relacionadas com a morte comuns à Ficha Confidencial de Notificação de Óbitos de Mulheres em Idade Fértil (ficha nova), obtida por meio da reinvestigação, à ficha antiga de notificação obtida por meio da investigação de rotina e à declaração de óbito. Camaragibe, Pernambuco, Brasil, 2000*.

\begin{tabular}{|c|c|c|c|c|c|c|}
\hline \multirow{2}{*}{$\begin{array}{l}\text { Procedimentos de investigação/ } \\
\text { proporções }\end{array}$} & \multicolumn{2}{|c|}{ Local de ocorrência } & \multicolumn{2}{|c|}{ Momento da morte } & \multicolumn{2}{|c|}{ Classificação do óbito feminino } \\
\hline & $\mathrm{n}$ & $\%$ & $\mathrm{n}$ & $\%$ & $\mathrm{n}$ & $\%$ \\
\hline Ficha nova (a) & 44 & 100,0 & 44 & 100,0 & 9 & 100,0 \\
\hline Ficha antiga (b) & 43 & 97,7 & 38 & 88,0 & 6 & 66,6 \\
\hline Declaração de óbito (c) & 43 & 97,7 & 34 & 77,7 & 4 & 44,4 \\
\hline Razão (a/b) & \multicolumn{2}{|c|}{1,02} & \multicolumn{2}{|c|}{1,20} & \multicolumn{2}{|c|}{1,50} \\
\hline Razão (a/c) & \multicolumn{2}{|c|}{1,02} & \multicolumn{2}{|c|}{1,30} & \multicolumn{2}{|c|}{2.20} \\
\hline Razão (b/c) & \multicolumn{2}{|c|}{1,00} & \multicolumn{2}{|c|}{1,10} & \multicolumn{2}{|c|}{1,50} \\
\hline
\end{tabular}

$* n=44$. Três casos de morte materna ocorreram fora de 2000 .

Tabela 3

Grau de preenchimento das variáveis sócio-demográficas comuns à Ficha Confidencial de Notificação de Ó bitos de Mulheres em Idade Fértil (ficha nova), obtida por meio da reinvestigação e à declaração de óbito. Camaragibe, Pernambuco, Brasil, 2000*.

\begin{tabular}{|c|c|c|c|c|c|c|c|c|c|c|}
\hline \multirow{2}{*}{$\begin{array}{l}\text { Procedimentos de investigação/ } \\
\text { proporções }\end{array}$} & \multicolumn{2}{|c|}{ Idade } & \multicolumn{2}{|c|}{ Raça/Cor } & \multicolumn{2}{|c|}{ Escolaridade } & \multicolumn{2}{|c|}{ Situação conjugal } & \multicolumn{2}{|c|}{ O cupação } \\
\hline & $\mathrm{n}$ & $\%$ & $\mathrm{n}$ & $\%$ & $\mathrm{n}$ & $\%$ & $n$ & $\%$ & $\mathrm{n}$ & $\%$ \\
\hline Ficha nova (a) & 63 & 98,4 & 63 & 98,4 & 62 & 96,8 & 62 & 98,4 & 55 & 86,0 \\
\hline Declaração de óbito (b) & 64 & 100,0 & 32 & 51,0 & 24 & 38,0 & 50 & 78,1 & 52 & 81,3 \\
\hline Razão (a/b) & \multicolumn{2}{|c|}{0,98} & \multicolumn{2}{|c|}{1,96} & \multicolumn{2}{|c|}{2,54} & \multicolumn{2}{|c|}{1,25} & \multicolumn{2}{|c|}{1,05} \\
\hline
\end{tabular}

$* n=64$. Três casos de morte materna ocorreram fora de 2000 .

Grau de preenchimento das variáveis relacionadas com a morte comuns à Ficha Confidencial de Notificação de Ó bitos de Mulheres em Idade Fértil (ficha nova), obtida por meio da reinvestigação e à declaração de óbito.

Camaragibe, Pernambuco, Brasil, 2000*.

\begin{tabular}{|c|c|c|c|c|c|c|}
\hline \multirow{2}{*}{$\begin{array}{l}\text { Procedimentos de investigação/ } \\
\text { proporções }\end{array}$} & \multicolumn{2}{|c|}{ Local de ocorrência } & \multicolumn{2}{|c|}{ Momento da morte } & \multicolumn{2}{|c|}{ Classificação do óbito feminino } \\
\hline & $\mathrm{n}$ & $\%$ & $n$ & $\%$ & $\mathrm{n}$ & $\%$ \\
\hline Ficha nova (a) & 64 & 100,0 & 64 & 100,0 & 10 & 100,0 \\
\hline Declaração de óbito (b) & 62 & 96,8 & 46 & 72,7 & 4 & 40,0 \\
\hline Razão (a/b) & \multicolumn{2}{|c|}{1,03} & \multicolumn{2}{|c|}{1,37} & \multicolumn{2}{|c|}{2,50} \\
\hline
\end{tabular}

$* n=64$. Três casos de morte materna ocorreram fora de 2000 . 
A baixa proporção do preenchimento da variável escolaridade observada no SIM nos dois momentos $(15,0 \%$ e $38 \%)$ confirma os resultados encontrados por Antunes (2001), para a população total de Pernambuco no período 1996/1998 (42\%). Neste sentido, é importante considerar que não foi avaliada a qualidade das informações destas variáveis, mas a capacidade de cada um dos procedimentos em registrá-las (expressa por meio das proporções e razões de proporções). Estudos baseados em informações pós-morte, obtidas de fontes secundárias como prontuários médicos ou laudos de necropsias ou, ainda, de fontes primárias como familiares e amigos, padecem de limitações inerentes à natureza das próprias fontes. A variável raça/cor é um bom exemplo da associação entre baixa cobertura e fidedignidade desconhecida, decorrentes da dificuldade de se lidar com as classificações de cor e raça e dos efeitos subliminares do racismo presentes no momento da população brasileira se autoclassificar.

A variável momento da morte foi melhor informada pelos dois procedimentos de investigação do que pelas informações da declaração de óbito. Esta variável foi incluída no modelo de declaração de óbito brasileiro em 1995, sob recomendação da OMS (Laurenti et al., 2000), no entanto, ainda exibe um elevado nível de subinformação. Um estudo recente conduzido no Brasil, mostrou que das 1.465 e 1.776 mortes maternas registradas no país em 1996 e
1997, aproximadamente $89 \%$ para 1996 e $87 \%$ para 1997, tiveram ignorado como resposta (Laurenti et al., 2000). No presente estudo, uma morte relacionada com a gravidez foi identificada entre as vinte mortes femininas não investigadas pelo sistema de vigilância de Camaragibe porque tinha resposta negativa à pergunta se estava ou esteve grávida no momento da morte, sendo reclassificada como morte materna tardia não declarada após investigação.

Os resultados demonstraram má qualidade das informações contidas na declaração de óbito, tanto no que diz respeito às variáveis sóciodemográficas como em relação às diretamente relacionadas com óbito. As diferenças entre mortes maternas declaradas e presumíveis, e mortes femininas não maternas estimadas por meio de diferentes procedimentos confirmam a subinformação da mortalidade materna já encontrada em Pernambuco e na cidade do Recife (Albuquerque, 1994; Valongueiro, 1996), a despeito de treinamentos e supervisões realizados pela Secretaria Estadual de Saúde de Pernambuco desde 1996, e por alguns municípios como Recife, Olinda, Camaragibe, Caruaru e Petrolina, com o apoio dos Comitês de Estudos da Mortalidade Materna. Tais resultados reforçam a necessidade de se monitorar os óbitos de mulheres em idade fértil, tendo como referência o método RAMOS, o procedimento que se mostrou mais acurado para identificar mortes maternas, e a nova ficha de notificação de óbitos de mulheres em idade fértil (Figura 1).

Figura 1

Ficha confidencial de notificação de óbito de mulheres em idade fértil-Q 1.

Informações obtidas da declaração de óbito e confirmadas através de investigação domiciliar.

SEÇÃO 0 - Dados Gerais

01 Instituição

Município

UF
Número da notificação

Data

Fonte

SEÇÃO 1 - Dados do caso

02 Nome

04 Idade
03 Endereço completo

Município
UF

(continua) 
Figura 1 (continuação)

$\begin{array}{ll}05 \text { Escolaridade (em anos de estudo) } & \\ \text { Nenhuma } & 0 \square \\ \text { De } 1 \text { a } 3 \text { anos } & 1 \square \\ \text { De } 4 \text { a } 7 \text { anos } & 2 \square \\ \text { De } 8 \text { a } 11 \text { anos } & 3 \square \\ \text { Mais de } 12 \text { anos } & 9 \square \\ \text { Sem informações } & 00 \square\end{array}$

07 Ocupação habitual

06 Situação Conjugal

Solteira

Casada

$1 \square$

Casad

Separada

União consensual

$\square$

$9 \square$

O utra

$5 \square$

Sem informações

$6 \square$

$00 \square$

08 Chefe de domicílio

Sim

$1 \square$

Não

$2 \square$

Sem informações

09 Raça/cor

Branca

$1 \square$

Indígena

$4 \square$

Negra

$2 \square$

Amarela

$5 \square$

Parda/morena

$3 \square$

Sem informações

$00 \square$

\section{SEÇÃO 2 - Dados do óbito}

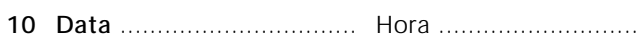

Número da DO

11 Local de ocorrência

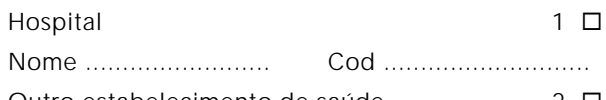

O utro estabelecimento de saúde $2 \square$

Domicílio

$3 \square$

Transporte/via pública

$4 \square$

Outro, qual

$5 \square$

Sem informações

$00 \square$

12 Momento do óbito

Gravidez $1 \square$

Aborto/pós-aborto $2 \square$

Puerpério (até 42 dias)

$4 \square$

Puerpério (de 43 dias a menos de um ano)

$5 \square$

Parto

$3 \square$

Não ocorreu durante estes períodos

Sem informações

$00 \square$

SEÇÃO 3 - Causas do óbito no atestado original
13 Causas da Morte
a) ........
b) .
c) .......
d)

CID

SEÇÃO 4 - Classificação inicial

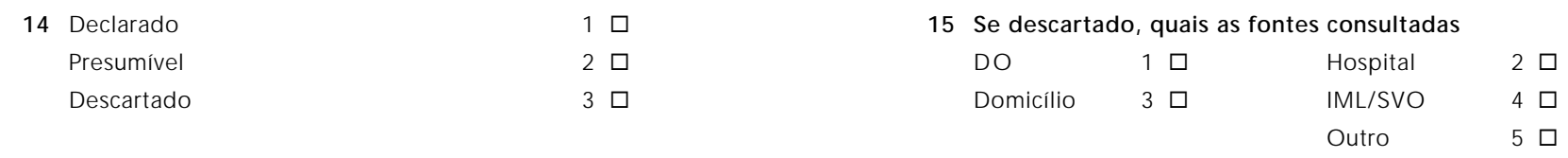

16 Observações do/a entrevistador/a

Data. 


\section{Referências}

ALBUQUERQUE, R., 1994. Estudo da Mortalidade Materna no Recife, 1992-1993. Dissertação de Mestrado, Campinas: Universidade de Campinas.

ANTUNES, M. B. C., 2001. Municipalização e Qualidade da Informação em Saúde. Dissertação de Mestrado, Recife: Programa Integrado de PósGraduação em Saúde Coletiva, Universidade Federal de Pernambuco.

IBGE (Fundação Instituto Brasileiro de Geografia e Estatística), 2001. Censo Demográfico. <http://www. ibge.gov.br>.

LAURENTI, R.; BUCHALLA, C. M.; LÓLIO, C. A.; SANTO, A. H. \& MELLO-JORGE, M. H. P., 1990. Mortalidade de mulheres em idade fértil no Município de São Paulo, 1986. I - Metodologia e resultados gerais. Revista de Saúde Pública, 24:128-133.

LAURENTI, R.; MELLO, J. M. H. \& GOTLIEB, S., 2000. Mortes maternas no Brasil: Análise do preenchimento da variável da declaração de óbito. Informe Epidemiológico do SUS, 9:43-50.

MS (Ministério da Saúde), 2001. Manual dos Comitês de Mortalidade Materna. 2a Ed. Brasília: MS.

NAM, C., 1990. Mortality differentials from a multiple cause of death perspective. In: Measurement and Analysis of Mortality (J. Vallin, S. D'Souza \& A. Palloni, ed.), pp. 328-342, New York: Oxford University Press.

OMS (Organização Mundial da Saúde), 1994. Classificação Estatística Internacional de Doenças e de Problemas Relacionados à Saúde. Décima Revisão, Manual de Instrução. v. 2. São Paulo: Centro Colaborador da OMS para Classificação de Doenças em Português.

PREFEITURA MUNICIPAL DE CAMARAGIBE, 2001. Boletim Epidemiológico 1993-2000. Camaragibe: Departamento de Epidemiologia e Vigilância Sanitária.
SES-PE (Secretaria Estadual de Saúde de Pernambuco), 1995. Portaria Estadual no 087/95. Diário Oficial do Estado de Pernambuco, 27 set.

SES-PE (Secretaria Estadual de Saúde de Pernambuco), 1999. Relatório de Investigação de Óbitos Maternos - 1997. Recife: Departamento de Vigilância Epidemiológica e Divisão de Saúde da Mulher.

TANAKA, A. C. \& MITSUIKI, L., 1999. Estudo da Magnitude da Mortalidade Materna em 15 Cidades Brasileiras. Relatório de Pesquisa. São Paulo: Faculdade de Saúde Pública, Universidade de São Paulo.

VALONGUEIRO, S. A., 1996. Mortalidade Materna em Pernambuco: Um Estudo Quantitativo e Qualitativo. Dissertação de Mestrado, Belo Horizonte: CEDEPLAR/Universidade Federal de Minas Gerais.

VOLOCHKO, A., 1992. Mortalidade de Mulheres Mortalidade Materna SUS-4, São Paulo. Dissertação de Mestrado, São Paulo: Faculdade de Saúde Pública, Universidade de São Paulo.

WHO (World Health Organization), 1994. Verbal Autopsies for Maternal Deaths. Report prepared on behalf of the Maternal Health and Safe Motherhood Programme. London: London School of Hygiene and Tropical Medicine.

WHO (World Health Organization), 2001. Maternal Mortality in 1995. Estimates Developed by WHO, UNICEF, UNFPA. Geneva: WHO.

Recebido em 3 de junho de 2003

Versão final reapresentada em 27 de agosto de 2003

Aprovado em 13 de outubro de 2003 